\title{
Stable Nonsupersymmetric Anti-de Sitter Vacua of Massive IIA Supergravity
}

\author{
Adolfo Guarino $\odot,{ }^{1,2, *}$ Emanuel Malek $\odot,{ }^{3, \dagger}$ and Henning Samtleben $\odot^{4, *}$ \\ ${ }^{1}$ Departamento de Física, Universidad de Oviedo, Avda. Federico García Lorca 18, 33007 Oviedo, Spain \\ ${ }^{2}$ Instituto Universitario de Ciencias y Tecnologías Espaciales de Asturias (ICTEA), Calle de la Independencia 13, 33004 Oviedo, Spain \\ ${ }^{3}$ Institut für Physik, Humboldt-Universität zu Berlin, IRIS Gebäude, Zum Großen Windkanal 6, 12489 Berlin, Germany \\ ${ }^{4}$ Univ Lyon, Ens de Lyon, Univ Claude Bernard, CNRS, Laboratoire de Physique, F-69342 Lyon, France
}

(Received 23 November 2020; accepted 4 January 2021; published 10 February 2021)

\begin{abstract}
Stable nonsupersymmetric anti-de Sitter (AdS) vacua of string theory are widely believed not to exist. In this Letter, we analytically compute the full bosonic Kaluza-Klein spectrum around the $G_{2}$-invariant nonsupersymmetric $\mathrm{AdS}_{4}$ solution of massive IIA supergravity and show that it is perturbatively stable. We also provide evidence that six other nonsupersymmetric $\mathrm{AdS}_{4}$ solutions of massive IIA supergravity are perturbatively stable. Since previous studies have indicated that these AdS vacua may also be nonperturbatively stable, our findings pose a challenge to the swampland conjecture.
\end{abstract}

DOI: 10.1103/PhysRevLett.126.061601

Constructing stable nonsupersymmetric vacua of string theory is one of its foremost challenges and crucial for applications to phenomenology, cosmology, and holography. Unlike their supersymmetric counterparts, such vacua are not protected by supersymmetry arguments and positive mass theorems [1]. The stability of nonsupersymmetric anti-de Sitter (AdS) vacua is particularly interesting, since they would provide a crucial tool for applying holography to realistic systems in condensed matter or indeed QCD. Moreover, nonsupersymmetric AdS vacua might be a stepping stone to understanding time-dependent de Sitter solutions in string theory.

The stability of nonsupersymmetric AdS vacua has proven particularly contentious in recent years. Various arguments have been put forward based on the weak gravity conjecture [2] that all nonsupersymmetric AdS vacua of string theory are unstable, ultimately leading to the AdS swampland conjecture [3]. Moreover, despite years of trying, no fully fledged case of a stable nonsupersymmetric AdS vacuum is known in string theory. Indeed, a powerful method for constructing nonsupersymmetric AdS vacua involves uplifting nonsupersymmetric solutions of lower-dimensional supergravities via a consistent truncation. However, all but a small number of these AdS solutions are already unstable within the lower-dimensional supergravity, with some of the scalar fields violating the Breitenlohner-Freedman (BF) bound [4]. Notable exceptions to this are the nonsupersymmetric $\mathrm{SO}(3) \times \mathrm{SO}(3)$ -

Published by the American Physical Society under the terms of the Creative Commons Attribution 4.0 International license. Further distribution of this work must maintain attribution to the author(s) and the published article's title, journal citation, and DOI. Funded by SCOAP. invariant $\mathrm{AdS}_{4}$ vacuum of four-dimensional $\mathcal{N}=8 \mathrm{SO}(8)$ gauged supergravity [5] obtained by consistent truncation of 11-dimensional supergravity on $S^{7}$ [6], and seven nonsupersymmetric $\mathrm{AdS}_{4}$ vacua of four-dimensional $\mathcal{N}=8$ ISO(7) gauged supergravity (see Appendix A of Ref. [7] for a summary and original references) obtained by consistent truncation of massive IIA supergravity on $S^{6}$ [8]. Remarkably, for these nonsupersymmetric $\mathrm{AdS}_{4}$ vacua, all 70 scalar fields in the $\mathcal{N}=8$ supergravity multiplet have masses above the BF bound [7,9].

Although these vacua are stable in four dimensions, their higher-dimensional stability is far from guaranteed. For example, the Kaluza-Klein (KK) spectrum around such vacua could contain tachyonic scalars violating the $\mathrm{BF}$ bound, or the AdS vacua may exhibit nonperturbative instabilities. Understanding the higher-dimensional stability has long been a challenge for these vacua, since both the computation of Kaluza-Klein spectra and the systematic search for nonperturbative instabilities (see, e.g., Ref. [10]) used to be notoriously difficult. However, within the last year, a new and powerful method based on exceptional field theory (ExFT) [11] was developed in Refs. [12,13] for computing Kaluza-Klein spectra of vacua of maximal gauged supergravities. At the same time, Ref. [14] showed that probe branes can be used to easily search for signals, dubbed brane-jet instabilities, of nonperturbative instabilities [10]. These new methods were used to show that the $\mathrm{SO}(3) \times \mathrm{SO}(3)$-invariant $\mathrm{AdS}_{4}$ vacuum has tachyonic Kaluza-Klein modes [15] and suffers from M2-brane-jet instabilities [14], with brane-jet instabilities also arising for other AdS vacua [16].

In this Letter, we will investigate the Kaluza-Klein spectrum of the seven nonsupersymmetric $\mathrm{AdS}_{4}$ vacua of the maximal $\mathrm{ISO}(7)$ supergravity, which correspond to 
compactifications of massive IIA supergravity on various deformed $S^{6}$ spheres and are stable within the fourdimensional supergravity. In particular, we focus on the vacuum with the largest bosonic symmetry, $G_{2}$. Remarkably, and in contrast to the $\mathrm{SO}(3) \times \mathrm{SO}(3)$ symmetric $\mathrm{AdS}_{4}$ vacuum of the $\mathrm{SO}(8)$ theory, the $\mathrm{AdS}_{4}$ vacuum with $G_{2}$ symmetry has shown to be $\mathrm{D} p$-brane-jet stable for $p=2,4,6,8$, while some other nonperturbative channels have also been ruled out [7]. Moreover, a partial search for the other six $\mathrm{AdS}_{4}$ vacua has also found no signs of D2-brane-jet instabilities [7]. Finally, because the compactification spaces have $S^{6}$ topology supported by fluxes, these vacua do not suffer from decays due to "bubbles of nothing" of the type argued in Ref. [17]. This suggests that these vacua may be nonperturbatively stable, making it even more important to study their perturbative KaluzaKlein stability in light of the weak gravity and swampland conjectures. This is what we set up to do in this Letter. In particular, we compute the full Kaluza-Klein spectrum of the $G_{2}$-invariant vacuum, providing the first analytic spectrum of a nonsupersymmetric vacuum, and use this to prove its perturbative stability. Moreover, we collect numerical evidence for the perturbative stability of the six other $\mathrm{AdS}_{4}$ vacua.

In Refs. [12,13], ExFT was used to derive mass matrices for the Kaluza-Klein spectrum around any vacuum of $\mathcal{N}=8$ gauged supergravities in four and five dimensions that arises from a consistent truncation of ten- or 11dimensional supergravity. Let us briefly review some of the salient features of ExFT and Kaluza-Klein spectroscopy.

ExFT is a reformulation of ten- or 11-dimensional supergravity, which unifies the metric and flux degrees of freedom within a manifestly $E_{7(7)}$ covariant formulation. Its bosonic sector

$$
\begin{aligned}
\left\{g_{\mu \nu}, \mathcal{M}_{M N}, \mathcal{A}_{\mu}{ }^{M}\right\}, & \mu=0, \ldots, 3, \\
& M=1, \ldots, 56
\end{aligned}
$$

consists of an external and an internal metric $g_{\mu \nu}, \mathcal{M}_{M N}$, respectively, with the latter parametrizing the coset space $E_{7(7)} / \mathrm{SU}(8)$, together with vector fields $\mathcal{A}_{\mu}{ }^{M}$ transforming in the $\mathbf{5 6}$ of the group $E_{7(7)}$.

As shown in Refs. [12,13], a general Kaluza-Klein fluctuation around a vacuum that uplifts from fourdimensional gauged supergravity can be expressed as a product of the modes of the consistent truncation, with a complete basis of functions on the compactification manifold. A powerful feature of this method is that this complete basis of functions can be chosen to be the scalar harmonics $\mathcal{Y}^{\Sigma}$ of the compactification with a metric that preserves the largest possible symmetry group $G_{\max }$ in the lowerdimensional gauged supergravity. In the case of the maximal ISO(7) supergravity investigated in this Letter, the internal space topology is $S^{6}$, the largest possible symmetry group is $G_{\max }=\mathrm{SO}(7)$, and we can choose the $\mathcal{Y}^{\Sigma}$ to be the scalar harmonics on the round $S^{6}$.

The fluctuation ansatz of the ExFT fields (1) around an $\mathrm{AdS}_{4}$ vacuum is given by $[12,13]$

$$
\begin{aligned}
g_{\mu \nu}(x, y) & =\rho^{-2}\left(\stackrel{\circ}{g}_{\mu \nu}(x)+\sum_{\Sigma} \mathcal{Y}^{\Sigma} h_{\mu \nu, \Sigma}(x)\right), \\
\mathcal{A}_{\mu}{ }^{M}(x, y) & =\rho^{-1}\left(U^{-1}\right)_{\underline{A}}{ }^{M} \sum_{\Sigma} \mathcal{Y}^{\Sigma} A_{\mu}{ }^{\underline{A}, \Sigma}(x), \\
\mathcal{M}_{M N}(x, y) & =U_{M^{\underline{A}}} U_{N^{-}}\left(\delta_{\underline{A} \underline{B}}+\mathcal{P}_{\underline{A} \underline{B}, I} \sum_{\Sigma} \mathcal{Y}^{\Sigma} j_{I, \Sigma}(x)\right),
\end{aligned}
$$

where the Kaluza-Klein fluctuations for the metric, vector fields, and scalars are labeled by $h_{\mu \nu, \Sigma}(x), A_{\mu}{ }^{\underline{A}, \Sigma}$, and $j_{I, \Sigma} \in \mathfrak{e}_{7(7)} \ominus \mathfrak{g} \mathfrak{u}(8)$, respectively. The latter appear under projection $\mathcal{P}_{\underline{A} \underline{B}, I}$, with $I=1, \ldots, 70$ resulting from the expansion of the group element $\mathcal{M}_{M N}$. On the other hand, $\rho(y) \in \mathbb{R}^{+}$and $U_{M}^{\underline{M}}(y) \in E_{7(7)}$ denote the scaling function and the twist matrix, respectively, encoding the consistent truncation to $\mathcal{N}=8$ gauged supergravity [18]. In the fluctuation ansatz (2), the twist matrix appears dressed with the scalar matrix of the four-dimensional supergravity $\mathcal{V}_{\underline{M}}{ }^{A} \in E_{7(7)} / \mathrm{SU}(8)$ evaluated at the scalar configuration specifying the vacuum of the maximal $D=4$ supergravity, i.e.,

$$
U_{M^{\underline{A}}}(y)=U_{M^{\underline{M}}}^{\underline{M}}(y) \mathcal{V}_{\underline{\underline{M}}}^{\underline{A}} .
$$

The fluctuation ansatz (2) induces mass matrices for the Kaluza-Klein spectrum which are entirely expressed through the embedding tensor of the $\mathcal{N}=8$ gauged supergravity $X_{\underline{M N}} \underline{P}$ and the linear action $\mathcal{T}_{\underline{M}}{ }^{\Sigma} \Omega$ of the $G_{\max }$ Killing vector fields on the scalar harmonics, both dressed by the scalar matrix $\mathcal{V}_{\underline{M}}{ }^{\underline{A}}$. The matrices $\mathcal{T}_{\underline{M}}{ }^{\Sigma} \Omega$ are explicitly defined as

$$
L_{\mathcal{K}_{\underline{M}}} \mathcal{Y}^{\Sigma}=-\mathcal{T}_{\underline{M}}{ }^{\Sigma}{ }_{\Omega} \mathcal{Y}^{\Omega},
$$

where $\mathcal{K}_{\underline{M}}$ are the Killing vectors generating $G_{\max }$, which can be extracted from the twist matrix $U_{M^{\underline{M}}}$. In turn, the dressed objects are defined as

$$
\begin{aligned}
& X_{\underline{A B}} \underline{\underline{C}}=\left(\mathcal{V}^{-1}\right)_{\underline{A}}^{\underline{M}}\left(\mathcal{V}^{-1}\right)_{\underline{B}}{ }^{\underline{N}} X_{\underline{M N}} \underline{\underline{P}} \mathcal{V}_{\underline{P}} \underline{C}, \\
& \mathcal{T}_{\underline{A}}{ }^{\Sigma} \Omega \\
& =\left(\mathcal{V}^{-1}\right)_{\underline{A}} \underline{M}_{\underline{M}} \mathcal{T}_{\Omega} .
\end{aligned}
$$

The mass matrices are obtained by linearizing the ExFT field equations with the fluctuation ansatz (2) [12,13]. For the purpose of this Letter, we give the scalar mass matrix in a yet more compact form: 


$$
\begin{aligned}
\mathcal{M}_{I \Sigma, J \Omega}^{(\text {scalar })}= & \mathcal{M}_{I J}^{(0)} \delta_{\Sigma \Omega}+\delta_{I J} \mathbb{M}_{\Sigma \Omega}^{(2)}+\mathcal{N}_{I J} \underline{\mathcal{T}}_{\underline{C}, \Sigma \Omega} \\
& -\frac{1}{6}\left(\Pi^{T} \Pi\right)_{I \Sigma, J \Omega} .
\end{aligned}
$$

Here, $\mathcal{M}_{I J}^{(0)}$ and $\mathbb{M}_{\Sigma \Omega}^{(2)}=-\left(\mathcal{T}_{A} \mathcal{T}_{A}\right)_{\Sigma \Omega}$ are the mass matrices of the four-dimensional supergravity scalars and of the spin-2 fluctuations, respectively. The matrices in the last two terms are given by

$$
\begin{aligned}
& \Pi_{\underline{A \Sigma, I \Omega}}=\delta_{\Sigma \Omega} X_{\underline{A C}^{\underline{D}}} \underline{\mathcal{P}}_{\underline{C D}, I}-12 \mathcal{P}_{\underline{A D, I}} \mathcal{T}_{\underline{D} \Omega}{ }^{\Sigma}, \\
& \mathcal{N}_{I J} \underline{C}=-4\left(X_{\underline{C A}}^{\underline{B}}+12 X_{\underline{A B}} \underline{C}\right) \mathcal{P}_{\underline{A} \underline{\underline{D}}}{ }^{[I} \mathcal{P}_{\underline{B}} \underline{D}^{J]} .
\end{aligned}
$$

In particular, the matrix $\Pi_{\underline{A} \Sigma, I \Omega}$ features in the linearized covariant scalar derivatives, $\partial_{\mu} j_{I, \Sigma}-\mathcal{A}_{\mu} \underline{\underline{C}} \Pi_{\underline{C} \Omega, I \Sigma}$ and thus encodes the projection onto the Goldstone scalars. Accordingly, it is orthogonal to the mass matrix and encodes the vector mass matrix as $\mathcal{M}^{\text {(vector) }}=\Pi \Pi^{T}$.

Let us now specialize to the specific ISO(7) gauged supergravity whose vacua we are interested in. In terms of $\mathrm{SL}(7) \subset E_{7(7)}$ defined by the branching of the $\mathbf{5 6}$ as

$$
A^{\underline{M}} \rightarrow\left\{A^{[a b]}, A^{a 8}, A_{[a b]}, A_{a 8}\right\},
$$

with $a=1, \ldots, 7$, the only nonzero components of the embedding tensor are

$$
X_{\underline{M} \underline{N}^{\underline{P}}}=\left\{\begin{array}{l}
X_{a b c d}{ }^{e f}=-X_{a b}{ }^{e f}{ }_{c d}=-8 \delta_{[a}^{[e} \delta_{b][c} \delta_{d]}^{f]}, \\
X_{a b c 8}{ }^{d 8}=-X_{a b}{ }^{d 8}{ }_{c 8}=-2 \delta_{[a}^{d} \delta_{b] c}, \\
X_{c 8 a b}{ }^{d 8}=-X_{c 8}{ }^{d 8}{ }_{a b}=-2 \delta_{c[a} \delta_{b]}^{d}, \\
X^{c 8}{ }_{a b}^{d 8}=-X^{c 8 d 8}{ }_{a b}=2 c \delta_{[a}^{c} \delta_{b]}^{d} .
\end{array}\right.
$$

Here, $c$ is a magnetic parameter that renders the gauging of dyonic type $[19,20]$ and which is identified with the Romans mass parameter [21] in ten dimensions.

For the fluctuation ansatz, we will be using the scalar harmonics on the round $S^{6}$, which can be expressed as symmetric traceless polynomials in the elementary harmonics $\mathcal{Y}^{a}$ satisfying $\mathcal{Y}^{a} \mathcal{Y}^{b} \delta_{a b}=1$. These are given by

$$
\mathcal{Y}^{\Sigma}=\left\{1, \mathcal{Y}^{a}, \mathcal{Y}^{a_{1} a_{2}}, \ldots, \mathcal{Y}^{a_{1} \ldots a_{\ell}}, \ldots\right\},
$$

with $\mathcal{Y}^{a_{1} \ldots a_{\ell}} \equiv \mathcal{Y}^{\left(\left(a_{1}\right.\right.} \ldots \mathcal{Y}^{\left.\left.a_{\ell}\right)\right)}$ and $(())$ denotes traceless symmetrization. Therefore, the $\mathcal{T}_{\underline{M}}{ }^{\Sigma} \Omega$ now simply become the $\mathrm{SO}(7)$ generators in the $[\ell, 0, \overline{0}]$ representation, with the only nonzero components given by

$$
\left.\mathcal{T}_{a b}{ }^{c_{1} \ldots c_{\ell}}{ }_{d_{1} \ldots d_{\ell}}=4 \ell \delta_{[a}{ }^{\left(\left(c_{1}\right.\right.} \delta_{b]\left(\left(d_{1}\right.\right.} \delta_{d_{2}}{ }^{c_{2}} \ldots \delta_{\left.d_{\ell}\right)}\right)^{\left.\left.c_{\ell}\right)\right)} .
$$

We stress once more that the harmonics (10) constitute an appropriate basis for the analysis of all vacua of this theory, regardless of the specific $S^{6}$ metric occurring at the vacua. With the data in Eqs. (9) and (11) specific to the maximal
ISO(7) supergravity, we can now proceed and compute the KK spectrum of the metric, vector, and scalar fluctuations around a given $\mathrm{AdS}_{4}$ vacuum specified by the scalar matrix $\mathcal{V}_{\underline{M}^{\underline{A}}}$ via the dressed tensors in Eq. (5).

The maximal ISO(7) supergravity possesses a rich structure of $\mathrm{AdS}_{4}$ vacua. To date, 60 such vacua have been identified up to discrete degeneracies (see Appendix A of Ref. [7]), all of which are contained within a $\mathbb{Z}_{2}^{3}$-invariant sector of the theory [22]. This sector describes an $\mathcal{N}=1$ supergravity coupled to seven chiral multiplets with complex scalar components $z_{i}$ $(i=1, \ldots, 7)$. Thus, the 56-bein entering the fluctuation ansatz (2) via the dressed twist matrix in Eq. (3) parametrizes a coset subspace

$$
\mathcal{V}_{\underline{M}^{A}}{ }^{A}\left(z_{i}\right) \in[\mathrm{SL}(2) / \mathrm{SO}(2)]^{7} \subset E_{7(7)} / \mathrm{SU}(8) .
$$

The Kähler potential $K\left(z_{i}, \bar{z}_{i}\right)$ and holomorphic superpotential $W\left(z_{i}\right)$ for the complex $z_{i}$ are given by

$$
\begin{aligned}
K\left(z_{i}, \bar{z}_{i}\right)= & -\sum_{i=1}^{7} \log \left[-i\left(z_{i}-\bar{z}_{i}\right)\right], \\
W\left(z_{i}\right)= & 2 g\left[z_{1} z_{2} z_{3}+z_{1} z_{6} z_{7}+z_{2} z_{5} z_{7}+z_{3} z_{5} z_{6}\right. \\
& \left.+\left(z_{1} z_{5}+z_{2} z_{6}+z_{3} z_{7}\right) z_{4}+c\right],
\end{aligned}
$$

where $g$ is the coupling constant in the four-dimensional supergravity that relates to the (inverse) radius of $S^{6}$.

This truncated sector contains seven nonsupersymmetric $\mathrm{AdS}_{4}$ vacua, yet stable within $D=4$ supergravity. I.e., all 70 scalars from the $\mathcal{N}=8$ supergravity multiplet have masses above the BF bound. At these vacua, the ISO(7) symmetry of the maximal theory is broken to $G_{\text {res }}$ [23-25], and the mass spectra organize into representations of $G_{\text {res }}$.

Employing the techniques reviewed above, we now compute the full bosonic Kaluza-Klein spectra around these nonsupersymmetric $\mathrm{AdS}_{4}$ vacua. Let us first focus on the $G_{2}$-invariant vacuum located at

$$
z_{1,2,3,4,5,6,7}=2^{-\frac{4}{3}}(-1+\sqrt{3} i) c^{\frac{1}{3}},
$$

with AdS radius $L^{2}=2^{-\frac{10}{3}} 3^{\frac{3}{2}} g^{-2} c^{\frac{1}{3}}$, and which was first constructed directly in ten dimensions in Ref. [26]. The mass spectrum of bosonic fields in the maximal supergravity was presented in Ref. [23], and a first rudimentary study of its higher-dimensional stability was performed in Ref. [27]. Here we will compute its full bosonic KaluzaKlein spectrum by exploiting the large residual symmetry group $G_{2}$ to analytically evaluate the mass matrices (6).

The normalized spin-2 mass matrix reduces to the $G_{2}$ Casimir operator

$$
L^{2} \mathbb{M}_{\Sigma \Omega}^{(2)}=-L^{2}\left(\mathcal{T}_{\underline{A}} \mathcal{T}_{\underline{A}}\right)_{\Sigma \Omega}=\frac{3}{2}\left(\operatorname{Cas}_{G_{2}}\right)_{\Sigma \Omega}
$$



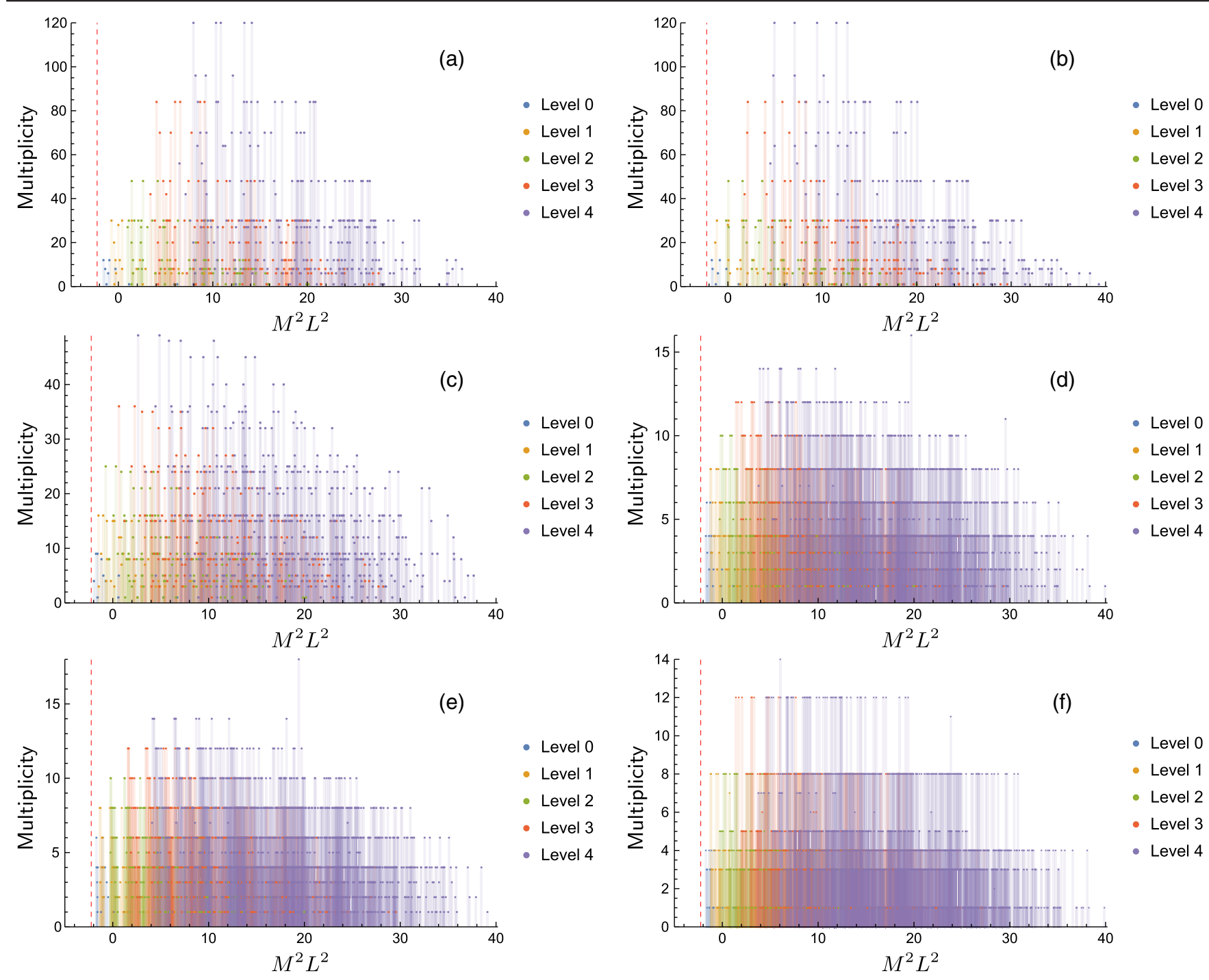

FIG. 1. Normalized masses $M^{2} L^{2}$ and multiplicities for the first four KK levels of spin-0 fluctuations around the six nonsupersymmetric and BF stable $\mathrm{AdS}_{4} \times S^{6}$ solutions of massive IIA supergravity given in Table I. The subfigure labels (a)-(f) correspond to the ID's of the different vacua in Table I. The dashed red line marks the BF bound $M^{2} L^{2}=-\frac{9}{4}$.

whose eigenvalues are given by [28]

$$
C_{\left[n_{1}, n_{2}\right]}=\frac{1}{3}\left(5 n_{1}+n_{1}^{2}+9 n_{2}+3 n_{1} n_{2}+3 n_{2}^{2}\right)
$$

on the $\left[n_{1}, n_{2}\right]$ representation. The spin-2 fluctuations at level $\ell$ transform in the $[\ell, 0]$ representation, which gives the mass eigenvalues $\frac{1}{2} \ell(\ell+5)$, in accordance with the results in Ref. [29].

For the scalar Kaluza-Klein modes, the first three terms of the (normalized) mass matrix (6) can be shown to combine into

$$
6 \delta_{I J} \delta_{\Sigma \Omega}+3\left(\operatorname{Cas}_{G_{2}}\right)_{\Sigma \Omega} \delta_{I J}-\frac{3}{2}\left(\operatorname{Cas}_{G_{2}}\right)_{I \Sigma, J \Omega},
$$

where the two Casimir operators act on the representations of the spin-2 harmonics and on the scalar fluctuations $j_{I, \Sigma}$, respectively. In contrast, the last term in Eq. (6) only affects the Goldstone scalars and ensures that these appear with zero eigenvalues. Putting this together with Eq. (16), we find that the normalized scalar masses at level $\ell$ allow for a surprisingly compact expression in terms of the quadratic $G_{2}$ Casimir eigenvalues as

$$
M_{\left[n_{1}, n_{2}\right]_{\ell}}^{2} L^{2}=(\ell+2)(\ell+3)-\frac{3}{2} C_{\left[n_{1}, n_{2}\right]},
$$

where $\left[n_{1}, n_{2}\right]_{\ell}$ denotes the $G_{2}$ representation that the Kaluza-Klein modes at level $\ell$ appear in. The form of the scalar mass matrix (6) implies that this mass formula also extends to the spin-1 sector. In particular, when evaluated for the first two levels, it reproduces the vector masses computed in Ref. [30]. 
TABLE I. Identifier, residual symmetry group, location, and AdS radius of the six nonsupersymmetric and BF-stable $\mathrm{AdS}_{4}$ vacua of the maximal $\mathrm{ISO}(7)$ supergravity with $\mathrm{SU}(3), \mathrm{SO}(4), \mathrm{U}(1) \times \mathrm{SO}(3)$, and $\mathrm{SO}(3)$ symmetry.

\begin{tabular}{lcrr}
\hline \hline ID & $G_{\text {res }}$ & $c^{-\frac{1}{3}} z_{i}$ & $g^{2} c^{-\frac{1}{3}} L^{2}$ \\
\hline (a) & $\mathrm{SU}(3)$ & $z_{1,2,3}=-0.2698+0.7329 i, z_{4,5,6,7}=0.4915+0.6618 i$ & 0.512522 \\
(b) & $\mathrm{SU}(3)$ & $z_{1,2,3}=-0.4547+0.8376 i, z_{4,5,6,7}=0.3348+0.6012 i$ & 0.511579 \\
(c) & $\mathrm{SO}(4)$ & $z_{1,2,3,5,6,7}=-0.4123+0.6512 i, z_{4}=-0.0678+1.1466 i$ & 0.510363 \\
(d) & $\mathrm{U}(1) \times \mathrm{SO}(3)$ & $z_{1,7}=-0.5061+0.7328 i, z_{2,3,5,6}=-0.3520+0.6240 i, z_{4}=-0.2732+0.9609 i$ & 0.511595 \\
(e) & $\mathrm{U}(1) \times \mathrm{SO}(3)$ & $z_{1,7}=-0.4869+0.7937 i, z_{2,3,5,6}=-0.3382+0.6059 i, z_{4}=-0.3736+0.9082 i$ & 0.511536 \\
(f) & $\mathrm{SO}(3)$ & $z_{1,7}=-0.5065+0.7285 i, z_{2,6}=-0.3663+0.6256 i,, z_{3,5}=-0.3403+0.6259 i$, & 0.511594 \\
& & $z_{4}=-0.2671+0.9623 i$ & \\
\hline \hline
\end{tabular}

For the spin-0 Kaluza-Klein modes, the relevant representations at level $\ell$ are the product of those of level 0 , $2([2,0] \oplus[1,0] \oplus[0,0])$, with $[\ell, 0]$, taking care to remove the Goldstone bosons. For generic $\ell$, this gives

$$
\begin{aligned}
& 2[\ell-4,2] \oplus[\ell-3,1] \oplus[\ell-3,2] \oplus 2[\ell-2,0] \\
& \oplus 3[\ell-2,1] \oplus 2[\ell-2,2] \oplus[\ell-1,0] \\
& \oplus 3[\ell-1,1] \oplus 5[\ell, 0] \oplus[\ell, 1] \\
& \oplus[\ell+1,0] \oplus 2[\ell+2,0],
\end{aligned}
$$

with care needed for the degeneracies at low $\ell$. Similarly, the spin-1 Kaluza-Klein modes at generic level $\ell$ transform in the representations

$$
\begin{array}{r}
{[\ell-3,1] \oplus[\ell-3,2] \oplus 3[\ell-2,1] \oplus 3[\ell-1,0]} \\
\oplus 3[\ell-1,1] \oplus 2[\ell, 0] \oplus[\ell, 1] \oplus 3[\ell+1,0],
\end{array}
$$

again with care needed for the degeneracies at low $\ell$. Evaluation of the mass formula (18) on the list of $G_{2}$ representations in Eq. (19) for the tower of spin-0 KaluzaKlein modes shows that the masses increase with increasing Kaluza-Klein level $\ell$ and are positive. This proves the perturbative stability of the nonsupersymmetric $G_{2}$ vacuum.

We finally turn to the other six nonsupersymmetric $\mathrm{AdS}_{4}$ solutions of massive IIA supergravity with smaller $G_{\text {res }}$, which correspond to compactifications of massive IIA supergravity on various deformed $S^{6}$ spheres and are summarized in Table I. The small residual symmetry groups $G_{\text {res }}$ do not allow us (for the moment) to analytically resolve these mass spectra. Instead, we revert to a numerical evaluation of the mass matrices (6) up to and including KK level $\ell=4$, with results displayed in Fig. 1. Remarkably, the analysis shows that all scalars on these levels are perturbatively stable with their normalized masses lying above the BF bound. Moreover, as is pictured in Fig. 1, the lowest-lying normalized masses at each Kaluza-Klein level $\ell$ increase monotonically with the level, thus suggesting that the full spectra will also be stable.

To summarize, we have analytically computed the full Kaluza-Klein spectrum of the nonsupersymmetric and
$G_{2}$-invariant $\mathrm{AdS}_{4} \times S^{6}$ background of massive IIA supergravity, resulting in the closed mass formula (18) encoding the entire scalar spectrum. This spectrum does not contain masses below the BF bound at any level in the KK tower of spin-0 fluctuations. Therefore, it presents an example of a nonsupersymmetric, yet perturbatively stable, solution of ten-dimensional massive IIA supergravity. We recall that this solution is a well-defined background of massive IIA string theory [8,31], and that the analysis of Ref. [7] has moreover excluded $\mathrm{D} p$-brane-jet instabilities for $p=2,4$, 6 , 8. We have also presented evidence that six other nonsupersymmetric $\mathrm{AdS}_{4} \times S^{6}$ backgrounds of massive IIA supergravity with smaller residual symmetry are also perturbatively stable in ten dimensions.

Since these $\mathrm{AdS}_{4}$ vacua have already passed a number of nontrivial tests regarding their nonperturbative stability, they seem to provide counterexamples to the belief that there are no stable nonsupersymmetric AdS vacua of string theory. Therefore, our findings here challenge the AdS swampland conjecture [3]. If the swampland conjecture is to hold, there must be an alternative decay channel for these nonsupersymmetric $\mathrm{AdS}_{4}$ vacua, which it would be imperative to unearth.

We would like to thank David Andriot and Davide Cassani for helpful discussions. A. G. is supported by the Spanish Government Grant No. PGC2018-096894-B-100 and by the Principado de Asturias through Grant No. FC-GRUPIN-IDI/2018/000174. E. M. is supported by the Deutsche Forschungsgemeinschaft (DFG, German Research Foundation) via the Emmy Noether program "Exploring the landscape of string theory flux vacua using exceptional field theory" (Project No. 426510644).

*adolfo.guarino@uniovi.es

†emanuel.malek@physik.hu-berlin.de

thenning.samtleben@ens-lyon.fr

[1] G. Gibbons, C. Hull, and N. Warner, The stability of gauged supergravity, Nucl. Phys. B218, 173 (1983).

[2] N. Arkani-Hamed, L. Motl, A. Nicolis, and C. Vafa, The string landscape, black holes and gravity as the weakest force, J. High Energy Phys. 06 (2007) 060. 
[3] H. Ooguri and C. Vafa, Non-supersymmetric AdS and the swampland, Adv. Theor. Math. Phys. 21, 1787 (2017).

[4] P. Breitenlohner and D.Z. Freedman, Stability in gauged extended supergravity, Ann. Phys. (N.Y.) 144, 249 (1982).

[5] N. Warner, Some new extrema of the scalar potential of gauged $N=8$ supergravity, Phys. Lett. 128B, 169 (1983).

[6] B. de Wit and H. Nicolai, $N=8$ supergravity, Nucl. Phys. B208, 323 (1982).

[7] A. Guarino, J. Tarrio, and O. Varela, Brane-jet stability of non-supersymmetric AdS vacua, J. High Energy Phys. 09 (2020) 110.

[8] A. Guarino, D. L. Jafferis, and O. Varela, The String Origin of Dyonic $N=8$ Supergravity and Its Simple ChernSimons Duals, Phys. Rev. Lett. 115, 091601 (2015).

[9] T. Fischbacher, K. Pilch, and N.P. Warner, New supersymmetric and stable, non-supersymmetric phases in supergravity and holographic field theory, arXiv:1010.4910.

[10] F. Apruzzi, G. B. De Luca, A. Gnecchi, G. Lo Monaco, and A. Tomasiello, On $\mathrm{AdS}_{7}$ stability, J. High Energy Phys. 07 (2020) 033.

[11] O. Hohm and H. Samtleben, Exceptional Form of $D=11$ Supergravity, Phys. Rev. Lett. 111, 231601 (2013).

[12] E. Malek and H. Samtleben, Kaluza-Klein Spectrometry for Supergravity, Phys. Rev. Lett. 124, 101601 (2020).

[13] E. Malek and H. Samtleben, Kaluza-Klein spectrometry from exceptional field theory, Phys. Rev. D 102, 106016 (2020).

[14] I. Bena, K. Pilch, and N. P. Warner, Brane-jet instabilities, J. High Energy Phys. 10 (2020) 091.

[15] E. Malek, H. Nicolai, and H. Samtleben, Tachyonic KaluzaKlein modes and the AdS swampland conjecture, J. High Energy Phys. 08 (2020) 159.

[16] M. Suh, The non-SUSY $\mathrm{AdS}_{6}$ and $\mathrm{AdS}_{7}$ fixed points are brane-jet unstable, J. High Energy Phys. 10 (2020) 010.
[17] H. Ooguri and L. Spodyneiko, New Kaluza-Klein instantons and the decay of AdS vacua, Phys. Rev. D 96, 026016 (2017).

[18] O. Hohm and H. Samtleben, Consistent Kaluza-Klein truncations via exceptional field theory, J. High Energy Phys. 01 (2015) 131.

[19] B. de Wit, H. Samtleben, and M. Trigiante, Magnetic charges in local field theory, J. High Energy Phys. 09 (2005) 016.

[20] G. Dall'Agata, G. Inverso, and A. Marrani, Symplectic deformations of gauged maximal supergravity, J. High Energy Phys. 07 (2014) 133.

[21] L. Romans, Massive $N=2 a$ supergravity in ten dimensions, Phys. Lett. 169B, 374 (1986).

[22] A. Guarino, J. Tarrio, and O. Varela, Flowing to $\mathcal{N}=3$ ChernSimons-matter theory, J. High Energy Phys. 03 (2020) 100.

[23] A. Borghese, A. Guarino, and D. Roest, All $G_{2}$ invariant critical points of maximal supergravity, J. High Energy Phys. 12 (2012) 108.

[24] A. Guarino and O. Varela, Dyonic ISO(7) supergravity and the duality hierarchy, J. High Energy Phys. 02 (2016) 079.

[25] A. Guarino, J. Tarrío, and O. Varela, Halving ISO(7) supergravity, J. High Energy Phys. 11 (2019) 143.

[26] D. Lust, F. Marchesano, L. Martucci, and D. Tsimpis, Generalized non-supersymmetric flux vacua, J. High Energy Phys. 11 (2008) 021.

[27] D. Cassani and A.-K. Kashani-Poor, Exploiting $N=2$ in consistent coset reductions of type IIA, Nucl. Phys. B817, 25 (2009).

[28] S. Okubo, Casimir invariants and vector operators in simple Lie algebra, J. Math. Phys. (N.Y.) 18, 2382 (1977).

[29] Y. Pang, J. Rong, and O. Varela, Spectrum universality properties of holographic Chern-Simons theories, J. High Energy Phys. 01 (2018) 061.

[30] O. Varela, Super-Chern-Simons spectra from exceptional field theory, arXiv:2010.09743.

[31] O. Varela, $\mathrm{AdS}_{4}$ solutions of massive IIA from dyonic ISO(7) supergravity, J. High Energy Phys. 03 (2016) 071. 